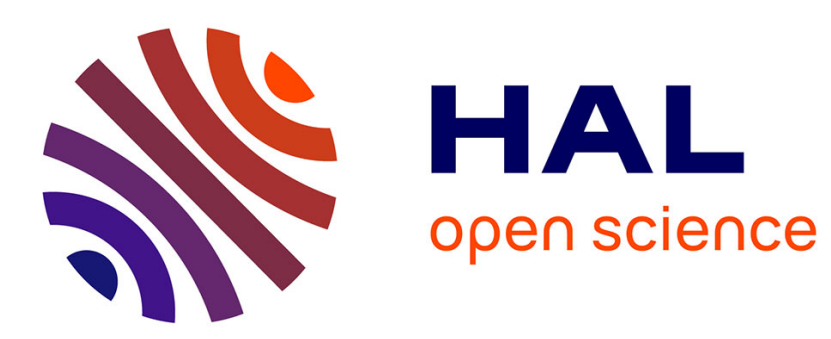

\title{
Z-relation and homometry in musical distributions
}

John Mandereau, Daniele Ghisi, Emmanuel Amiot, Moreno Andreatta, Carlos

Agon

\section{To cite this version:}

John Mandereau, Daniele Ghisi, Emmanuel Amiot, Moreno Andreatta, Carlos Agon. Z-relation and homometry in musical distributions. Journal of Mathematics and Music, 2011, 5 (2), pp.83-98. 10.1080/17459737.2011.608819 . hal-00664776

\section{HAL Id: hal-00664776 \\ https://hal.science/hal-00664776}

Submitted on 31 Jan 2012

HAL is a multi-disciplinary open access archive for the deposit and dissemination of scientific research documents, whether they are published or not. The documents may come from teaching and research institutions in France or abroad, or from public or private research centers.
L'archive ouverte pluridisciplinaire HAL, est destinée au dépôt et à la diffusion de documents scientifiques de niveau recherche, publiés ou non, émanant des établissements d'enseignement et de recherche français ou étrangers, des laboratoires publics ou privés. 


\title{
Z-Relation and Homometry in Musical Distributions
}

\author{
John Mandereau ${ }^{\mathrm{a}, \mathrm{d} * *}$, Daniele Ghisi ${ }^{\mathrm{b}}$, Emmanuel Amiot ${ }^{\mathrm{c}}$, \\ Moreno Andreatta ${ }^{\mathrm{d}}$ and Carlos Agon $^{\mathrm{d}}$ \\ a Dipartimento di Matematica, Università di Pisa, Italy; ${ }^{\mathrm{b}}$ Cursus de composition, \\ IRCAM, Paris, France; ${ }^{\mathrm{c}}$ CPGE, Perpignan, France; d UMR 9912, \\ CNRS/IRCAM/UPMC, Paris, France
}

This is a preprint of an article whose final and definitive form has been published in the Journal of Mathematics and Music (JMM) (c) 2011 Taylor \& Francis.

The published article is available online at http://www.tandfonline.com/doi/abs/10.1080/17459737.2011.608819

\begin{abstract}
This paper defines homometry in the rather general case of locally-compact topological groups, and proposes new cases of its musical use. For several decades, homometry has raised interest in computational musicology and especially set-theoretical methods, and in an independent way and with different vocabulary in crystallography and other scientific areas. The link between these two approaches was only made recently, suggesting new interesting musical applications and opening new theoretical problems. We present some old and new results on homometry, and give perspective on future research assisted by computational methods. We assume from the reader basic knowledge of groups, topological groups, group algebras, group actions, Lebesgue integration, convolution products, and Fourier transform.
\end{abstract}

Keywords: GIS (Generalized Interval Systems); interval vector; Patterson function; Z-relation; homometry; hexachord theorem.

MCS/CCS/AMS Classification/CR Category numbers: AMS MSC 05E15, 20H15, $43 \mathrm{~A} 20$.

\section{Introduction}

Although already present in Howard Hanson's work [8], the concept of Z-relation is presented and discussed in a systematic way by Allen Forte in [6]. In the classical framework of musical set theory, the $n$-tone equal temperament is modeled via the cyclic group $\mathbb{Z}_{n}=\mathbb{Z} / n \mathbb{Z}$, and each class of $\mathbb{Z}_{n}$ is said to be a pitch-class. Any pitch-class set is simply called set ${ }^{1}$ For any set $A \subseteq \mathbb{Z}_{n}$ one can define the interval vector (iv) as for every $k \in \mathbb{Z}_{n}, \mathbf{i v}(A)_{k}=\operatorname{ifunc}(A, A)_{k}=\#\left\{(s, t) \in A^{2}, t-s=k\right\}$. One

\footnotetext{
** Corresponding author. Email: mandereau@mail.dm.unipi.it

${ }^{1}$ We denote any set $\left\{\left[a_{1}\right]_{n}, \ldots,\left[a_{s}\right]_{n}\right\}$ as $\left\{a_{1}, \ldots, a_{s}\right\}_{n}$.
} 


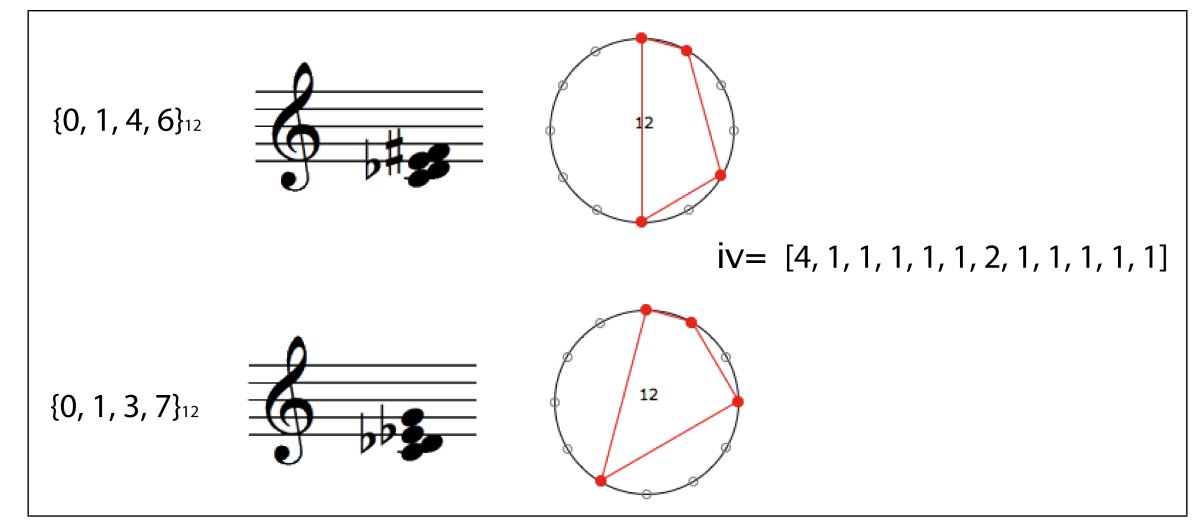

Figure 1: A well-known example of Z-related sets, in $\mathbb{Z}_{12}$.

might notice that we define the iv function via the ifunc function borrowed from David Lewin [11] Forte's original icv only features 6 values, because of inherent symmetries, e.g. for a diatonic scale the values of iv are $[7,2,5,4,3,6,2,6,3,4,5,2]$ and Forte only keeps $<2,5,4,3,6,1>$ (the tritone is only counted once and the cardinality $\mathrm{iv}(0)$ is ommited). Since we generalize the notion to much more complicated groups than $T$ (or $T / I$ ), and later to $k$-sets instead of couples of elements, it is convenient to keep the whole list instead of a reduced version. A brief history of the interval class vector is found in [5, Sec. 1.2].

Two sets $A$ and $B$ are said to be $Z$-related if $\mathbf{i v}(A) \equiv \mathbf{i v}(B)$, i.e. if the same number of intervals of each type is showing up in both sets. In other words, $A$ and $B$ share the same interval content. Clearly, transposing or inverting a set does not change its interval content, and thus we have a lot of trivially Z-related sets. In order to avoid this trivial case, we may consider sets classes up to transposition and inversion, and we notice that there still exists Z-related sets in Forte's sense ${ }^{2}$ A well-known example is sets $\{0,1,4,6\}_{12}$ and $\{0,1,3,7\}_{12}$ in $\mathbb{Z}_{12}$, which share the same interval vector $[4,1,1,1,1,1,2,1,1,1,1,1]$ - see Figure 1. Some composers have (implicitly or explicitly) dealt with the Z-relation; for example this couple of Z-related sets is exactly the one used by Elliot Carter in his second quartet [7].

To improve upon the classical model, one can substitute pitch-class sets with multisets, i.e. integer-valued distributions, which might be useful to represent a chord where notes might be repeated (Fig. 22, center); one can even consider rational- or real-valued distribution ${ }^{3}$, which include in the representation the dynamics of each note (Fig. 2, right). In this case, the interval vector is no more sufficient, and must be replaced (as we will see) by the Patterson function, which will extend the concept of interval content, as it represents (as suggested by Lewin) the probability of hearing a given interval, if the notes of a given set are played randomly.

The name Patterson function comes from X-ray crystallography. Let $G$ be an abelian group (with

2 i.e. unrelated by transposition or inversion. In [1] the full equivalence relation, including the trivial cases, is called Lewin's relation.

${ }^{3}$ Let $K$ be a field and let $G$ be an abelian group (with additive notation). A distribution on $G$ with coefficients in $K$ has the form $E=\sum_{g \in G} a_{g} \delta_{g}$, where $a_{g} \in K$ and $\delta_{g}$ is the Dirac mass related to the element $g$. For practical purposes a distribution can be viewed as the map $g \mapsto a_{g}$. Non integral values happen in many practical applications, say, for instance, the probability of occurrence of a given note, or interval, in a whole piece of music. If $a_{g} \neq 0$ only finitely often, we say that the distribution is finite. Recall that the algebra of such distributions under the convolution product is isomorphic with the group $\operatorname{ring} K^{G}$, and thus we will sometimes write $E \in K^{G}$. 


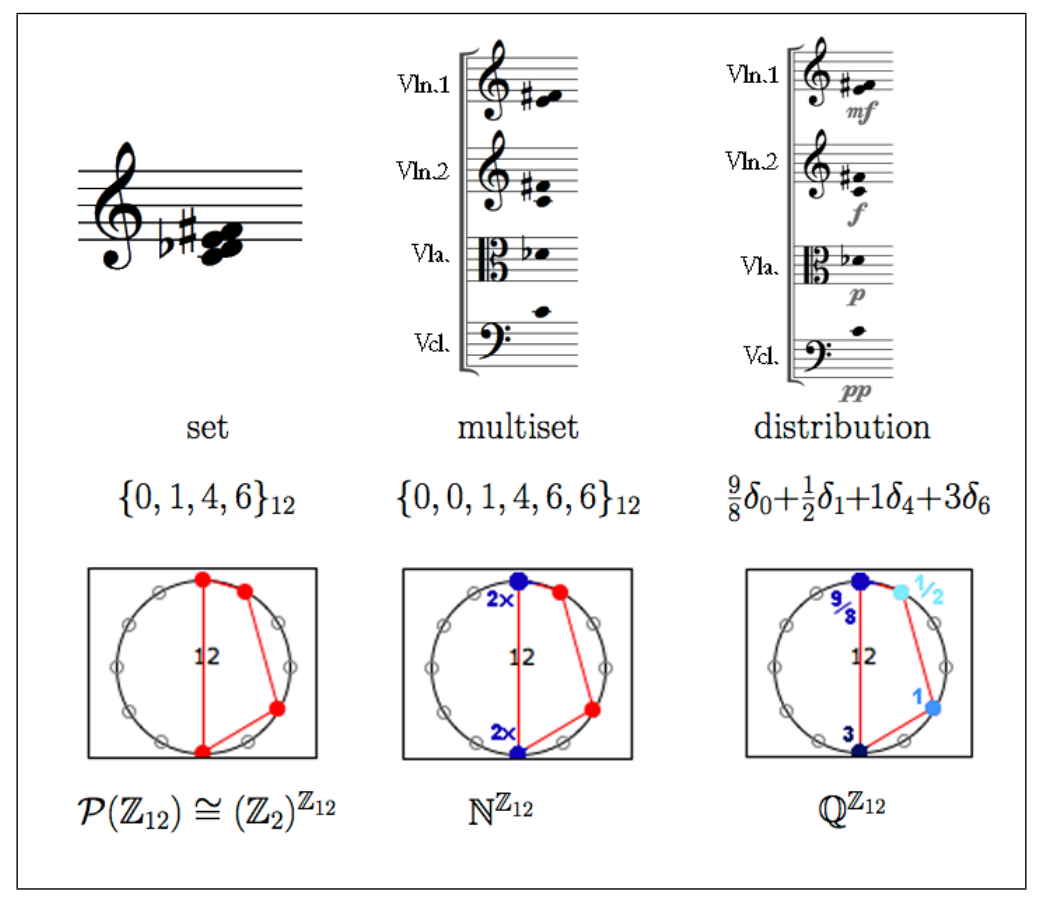

Figure 2: An example showing the usefulness of improving the classical model. A standard set is an element of $\mathcal{P}\left(\mathbb{Z}_{n}\right)$, i.e. a $0-1$ distribution on $\mathbb{Z}_{n}$. If we allow some notes to be repeated, we have a multiset, as in the middle example (the same chord given to a string quartet), i.e. a distribution of $\mathbb{N}^{\mathbb{Z}_{n}}$. Finally, if we add a dynamic mapping (right example), we can see the chord as a real distribution, i.e. a distribution of $\mathbb{Q}^{\mathbb{Z}_{n}}$. In this example we have arbitrarily chosen $m f=1, f=2$, $p=1 / 2, p p=1 / 4$. 
additive notation). Given a distribution $E=\sum_{g \in G} e_{g} \delta_{g}$, we call inversion ${ }^{2}$ of $E$ the distribution $I(E)=\sum_{g \in G} \bar{e}_{g} \delta_{-g}$, and the $k$-transposition of $E$ is the distribution $T_{k}(E)=\sum_{g \in G} e_{g} \delta_{g+k}(k \in G)$. Then, the Patterson function of any distribution $E$ is the convolution product $E * I(E)$. Now, for any $X \subseteq G$, let $\mathbf{1}_{X}$ be the distribution $\sum_{g \in X} \delta_{g}$. By reading [10, we know that $\mathbf{i v}(A)=\mathbf{1}_{A} * \mathbf{1}_{-A}$, and since $\mathbf{1}_{-A}=I\left(\mathbf{1}_{A}\right)$, we see that the Patterson function is nothing more than a generalization of the interval vector to a generic distribution. In crystallography, the Patterson function is the starting point for solving the phase retrieval problem, i.e. to determine the arrangement of atoms within a crystal, given the module of the Fourier transform ${ }^{3}$ of the atoms' distribution. Indeed, if we know $D * I(D)$, we know the absolute values of its Fourier transform $\widehat{D} \widehat{D}(\omega)=\|\widehat{D}(\omega)\|^{2}$ for all $\omega \in \mathbb{Z}_{n}$. Thus, to reconstruct $\widehat{D}(\omega)=\|\widehat{D}(\omega)\| e^{i \phi(\omega)}$ (and $D$ from there by inverse Fourier transform), since we know its module, we just need to retrieve the phase $\phi(\omega)$. This is the central problem that we address in this paper.

In this article, we will link vocabulary from musical set theory — Generalized Interval System, interval vector, Z-relation - with vocabulary from crystallography - implicit usage of group structure, Patterson function, homometry. These objects and their elementary properties are presented in a theoretical framework large enough to cover most of the areas wherein homometry and Z-relation have been previously studied. In Section 2, we introduce topological and measure and integration theory tools that we use on Lewin's Generalized Interval Systems (GIS); in Section 3 we introduce the interval content and the Patterson function, and in Section 4 Z-relation and homometry. Then, we study properties of Patterson functions and homometry: in Section 5 we relate interval structure and interval content, including two examples of a Z-relation in a non-commutative GIS; in Section 6 we study how Patterson functions transfer through quotients, and in Section 7 we present and illustrate a generalized hexachord theorem.

\section{Using Generalized Interval Systems (GIS)}

\subsection{Mathematical definition of a GIS}

The notion of Generalized Interval System, introduced in [11, formalizes the notion of interval between two points in a set of values of an abstract musical parameter.

Definition 2.1 (Lewin). A Generalized Interval System (GIS) is a triple ( $S, G$, int), where $S$ is a set called space of the GIS, $G$ a group called interval group of the GIS, and int : $S \times S \rightarrow G$ a map such that

(A) For every $r, s, t$ in $S$, int $(r, s) \operatorname{int}(s, t)=\operatorname{int}(r, t)$.

(B) For every $s$ in $S, i$ in $G$, there is a unique $t$ in $S$ such that $\operatorname{int}(s, t)=i$.

It is noted in [17 that

- (A) and (B) in the definition above are equivalent to defining a simply transitive right action of group $G$ on $S$, such that for every $s, t$ in $S, s \operatorname{int}(s, t)=t$;

\footnotetext{
${ }^{2}$ The inversion of $E$, namely $I(E)$, is sometimes found as $E^{\prime}$ or $E^{*}$ and referred to as reflection.

${ }^{3}$ Recall that, for $G=\mathbb{Z}_{n}$, the Fourier transform of a distribution $E=\sum_{g \in \mathbb{Z}_{n}} e_{g} \delta_{g}$ is the map

$$
\omega \in \mathbb{Z}_{n} \mapsto \widehat{E}(\omega)=\sum_{g \in \mathbb{Z}_{n}} e_{g} \exp (-2 i \pi g \omega / n)
$$
}


- the definition of a GIS is analogous with the definition of an affine space, the difference being that the underlying algebraic structure of an affine space is not a group, but a vector space.

In every GIS, the musical parameter space $S$ and the interval group $G$ have the same cardinality; more precisely, condition (B) implies that for every $s$ in $S$, the label man ${ }^{4}$ is bijective:

$$
\text { label : } \begin{aligned}
S & \rightarrow G \\
t & \mapsto \operatorname{int}(s, t)
\end{aligned}
$$

We develop now two usages of label bijections, which are also common with the couple "affine space-vector space".

The first possibility is using the interval group $G$ itself as the space $S$ : in this case, the group action that defines the GIS is right translation, i.e. for every $s, t$ in $G$, $\operatorname{int}(s, t)=s^{-1} t$. As a consequence, every group defines a canonical GIS associated with it via this group action. To avoid confusion that may arise from this identification of the interval group $G$ and the GIS space, elements of the space will be called points, elements of the interval group will be called intervals, and unless explicitly mentioned otherwise, subsets of $G$ mean subsets of the GIS space.

The second possibility is using label bijections for transferring some additional structure of the interval group $G$ - e.g. a topology, a distance or a measure - onto $S$. Moreover, if this structure is translation invariant, the resulting structure on $S$ does not depend on a particular $s \in S$ that defines label map. This principle of translation-invariant structure transfer for GIS is detailed in [9], and we will use it below.

When $G$ is abelian, we will denote the group operation with a plus sign + instead of a multiplicative notation. Although most of our examples will happen in the commutative case, the definition and several basic properties of the objects that we will define also hold in the non-abelian case. A musically significant example of a non-commutative GIS is the GIS of time spans[11, 4.1.3.1], which is defined as the positive affine group of $\mathbb{R}$, that is the semi-direct product $\mathbb{R} \rtimes_{m} \mathbb{R}_{+}^{*}$ where the group morphism $m:\left(\mathbb{R}_{+}^{*},.\right) \rightarrow(\operatorname{Aut}(\mathbb{R}),+)$ maps $r$ to multiplication by $r$.

\subsection{Transferring translation-invariant topologies and measures onto a GIS}

We are interested in measuring subsets of the space of a GIS. The most straightforward measure of a set is its cardinality; however, many definitions and tools we will present are, under some conditions, still valid with using certain measures - e.g. the Lebesgue measure - on a GIS. More precisely, we need a measure on both the space of a GIS and its interval group, and we require that the measure on the interval group be translation-invariant, so that the measure on the space naturally comes from transferring the measure of the group; we will implicitly assume from now on that defining a translation-stable $\sigma$-algebra $\mathcal{A}$ (the borelian subsets, see notations below) on a group $G$ and a measure on $\mathcal{A}$ also defines, through the transfer principle, the same structures on the space of a GIS with $G$ as its interval group. We will exclude structures which are not translation-invariant, because giving different weights to a subset and its translations would break the concept of an isotropic GIS with its transfer principle. This generalization of measuring the cardinality of sets in GIS has already been proposed by Lewin in [11, section 6.10], but has never been further elaborated as far as we know. We believe that such a generalization is not gratuitous, from a mathematical point of view.

\footnotetext{
${ }^{4}$ The denomination label comes from [11, beginning of Chap. 3].
} 
In fact, there are fortunately many groups which may be fitted with a right-translation-invariant measure, thanks to the following result.

Definition 2.2. Let $(G, \mathcal{A}, \mu)$ be a measured space where $G$ is a group. $\mu$ is called righttranslation-invariant if $\mathcal{A}$ is right-translation-stable and for every $A \in \mathcal{A}, g \in G, \mu(A g)=\mu(A)$. If, in addition, $G$ is a topological group, and $\mathcal{A}$ is the Borel $\sigma$-algebra on $G$, then $\mu$ is called a right Haar measure on $G$.

Theorem 2.3. Any locally-compact Hausdorff topological group $G$ has a right Haar measure $\mu$; moreover, this measure is uniquely defined, up to a multiplicative constant.

The previous theorem, which is a classical theorem in topology, allows us to define the notion of interval content in any locally-compact topologic group, including every group with the discrete topology - the associated right Haar measure is simply the cardinality function $-\mathbb{R}$, and all products and quotients of such groups.

Since the topology of a topologic group $G$ is translation-invariant, it can be naturally transferred onto the space of a GIS that has $G$ as its interval group. We recall the idea from [9], that using topologies in GIS could help express notions of continuity of musical patterns; this would make sense for instance with $\mathbb{R}$, the continuous circle $\mathbb{R} / \mathbb{Z}$, or any product of these groups fitted with their respective usual topologies, as an interval group of a GIS.

As we want to be able to compare measures of certain sets and to do some computations on measure values (multiplications, additions, subtractions...), we will restrict our study to measurable sets with finite measure, as suggested in [11].

We end this introduction of topological GIS with a (right) Haar measure with some notations, which we will assume throughout the rest of the article. Let $G$ be a locally compact group, $K$ a subfield of $\mathbb{C}$ closed under the complex conjugation $: x \mapsto \bar{x}$; we denote

- $S(X)$ the permutation group of a set $X$,

- $\mathcal{A}$ the $\sigma$-algebra of Borel sets of $G$,

- $\mu$ a right Haar measure on $G$,

- $\tilde{\mathcal{A}}$ the set of measurable subsets of $G$ with finite measure,

- $K^{G}$ the $K$-algebra of maps from $G$ to $K$, which are also called ( $K$-valued) distributions on $G$,

- for every $g \in G, T_{g}: K^{G} \rightarrow K^{G}$

$$
E \mapsto\left(T_{g}(E): h \mapsto E\left(g^{-1} h\right)\right)
$$

the left translation of distributions by $g$; we may also write $T_{g}(A)=g A$ for $A \subset G$ when there is no ambiguity;

- $T(G)=\left\{h \mapsto T_{g}(h)=g h, g \in G\right\}$, or simply $T$, the group of left translations on $G$,

- $I: K^{G} \rightarrow K^{G} \quad$ the inversion on distributions; we also overload $I$ by defin-

$$
E \mapsto\left(I(E): h \mapsto \overline{E\left(h^{-1}\right)}\right)
$$
ing, for every $A \subset G, I(A)=A^{-1}$,

- $D(G)$ (or $D$ ) the generalized dihedral group over $G$, which is the subgroup of $S(G)$ generated by the left translations of $G$ and the inversion $g \mapsto g^{-1}$, 
- $\mathcal{D}(G)$ or $\mathcal{D}$ the subgroup of the linear group of $K^{G}$ generated by $\left\{T_{g}, g \in G\right\} \cup\{I\}$, which is an isomorphic representation of $D(G)$,

- when $K \in\{\mathbb{R}, \mathbb{C}\}, \Sigma_{\mathrm{C}}(G, k)$ the algebra of bounded functions ${ }^{5}$ with compact support from $G$ to a subset $k$ of $K$; this is the class of functions on which we will define the Patterson function;

- $[x]_{H}=\{h(x), h \in H\}$ where $X$ is a set, $H$ a subgroup of $S(X)$ and $x \in X ;[x]_{H}$ is the orbit of $x$ under the natural group action of $H$ on $X$, elements of $[x]_{H}$ are said congruent to $x$ modulo $H$; the same notation is used with $H$ a subgroup of a group $G$ and for every $g \in G[g]_{H}=H g$;

- for every $a, b$ in $\mathbb{Z}, \llbracket a, b \rrbracket=\{x \in \mathbb{Z}, a \leqslant x \leqslant b\}$.

It should be noticed that, in $\operatorname{defining} \operatorname{int}(a, b)$ as $a^{-1} b$, we favor left translations over right translations: for any $a, b, c \in G$, one has $\operatorname{int}(c a, c b)=a^{-1} c^{-1} c b=a^{-1} b=\operatorname{int}(a b)$, but $\operatorname{int}(a c, b c)=$ $c^{-1} a^{-1} b c=c^{-1} \operatorname{int}(a, b) c \neq \operatorname{int}(a, b)$ in general. Thus this notion of interval is invariant by left translations only 6 There is, of course, an alternative definition of the interval from $a$ to $b$, namely $\widetilde{\operatorname{int}}(a, b)=b a^{-1}$, which is invariant under right translation. This explains why we have found not one, but two generalizations of the hexachord theorem (see subsection 7 below). Obviously, the abelian case is much simpler, with only one possible notion of interval, and one kind of translation. In the sequel, unless otherwise indicated, we keep with $\operatorname{int}(a, b)=a^{-1} b$.

In general, in a non-abelian locally compact group, the left- and right-invariant Haar measures do not coincide; for instance, in the affine group of maps $x \mapsto a x+b$ on the real line, the leftand right- invariant measures are respectively $d a d b / a^{2}$ and $d a d b / a$. This motivates the following definition.

Definition 2.4. A locally compact group is unimodular if it admits a Haar measure that is both right- and left-invariant.

The unimodularity is a reasonable assumption in many cases; in particular, it is satisfied whenever $G$ is compact - see [14, Chap. 3, 1(iv)] - and even more easily when $G$ is discrete - since cardinality is both right- and left-translation-invariant.

\section{Interval vector and Patterson function}

Definition 3.1. Let $A, B$ in $\tilde{\mathcal{A}}$. The interval function between $A$ and $B$ is the function

$$
\text { ifunc }(A, B): \quad \begin{aligned}
G & \rightarrow \mathbb{R}_{+} \\
g & \mapsto \mu(B \cap A g)
\end{aligned}
$$

Since $B \cap A g=\{a \in A, \exists b \in B, \operatorname{int}(a, b)=g\}$, this definition is a straightforward generalization of [11, 5.1.3], where ifunc is defined for discrete $G$.

Definition 3.2. Let $A \in \tilde{\mathcal{A}}$. The interval content of $A$ is the function

$$
\begin{aligned}
\operatorname{iv}(A): \quad & \rightarrow \mathbb{R}_{+} \\
g & \mapsto \mu(A \cap A g)
\end{aligned}
$$

\footnotetext{
${ }^{5}$ In measure theory, this should be read 'almost everywhere' as usual; for a definition, see [16] Def. 1.35].

6 This fact is well commented in [11, section 3.4].
} 
If $G$ is discrete, the interval content is also called interval vector, hence the notation iv.

It is clear, from the right translation invariance of $\mu$ and the fact that it is real-valued, that for every $A \in \tilde{\mathcal{A}}$ and $g \in G, \operatorname{iv}(A)(g)=\mu\left(A g^{-1} \cap A\right)=\overline{\mu\left(A g^{-1} \cap A\right)}$, i.e. $I(\mathbf{i v}(A))=\mathbf{i v}(A)$. In [10, the interval vector is expressed as a convolution product through the natural bijection between $\tilde{\mathcal{A}}$ and $\Sigma_{\mathrm{C}}(G,\{0,1\})$, i.e. $\mathbf{i v}(A)=\mathbf{1}_{A} * \mathbf{1}_{A^{-1}}$. However, to include the case of a non-commutative group, the interval content shall be expressed as $\mathbf{i v}(A)(g)=\int \mathbf{1}_{A}\left(h g^{-1}\right) \mathbf{1}_{A}(h) d \mu(h)=I\left(\mathbf{1}_{A}\right) * \mathbf{1}_{A}(g)$, where $*$ is the convolution product for the right Haar measure - see [14, Chap. 3, 3.5 and 5.1]. Then, this definition can be extended to every (almost everywhere) bounded function on $G$ with compact support, which is customary in crystallography; for example, see the introduction of [15].

In a non abelian group, we can introduce two distinct definitions of the interval content, because there are two different definitions of the interval from $a$ to $b$.

Definition 3.3. We note ${ }^{r}$ ifunc $=$ ifunc,${ }^{r} \mathbf{i v}=\mathbf{i v}$ the right interval function and interval content already defined above. Let ${ }^{l}$ ifunc $(A, B)$ be the left interval function:

$$
g \in G \mapsto{ }^{l} \text { ifunc }(A, B)=\mu(B \cap g A)=\int \mathbf{1}_{B}(h) \mathbf{1}_{A}\left(g^{-1} h\right) d \mu(h)
$$

Similarly the left interval content is defined as

$$
{ }^{l} \mathbf{i v}(A): g \in G \mapsto \mu(A \cap g A)=\int \mathbf{1}_{A}(h) \mathbf{1}_{A}\left(g^{-1} h\right) d \mu(h)
$$

Unless otherwise indicated, we will use the rightwise definitions of the interval function and interval content.

Definition 3.4. For every function $\left.E \in \Sigma_{\mathrm{C}}(G, K)\right]^{7}$ the Patterson function of $E$ is defined by

$$
d^{2}(E):=I(E) * E: g \in G \mapsto \int \overline{E\left(h g^{-1}\right)} E(h) d \mu(h)
$$

As the interval content of a finitely measured subset of $G$ is the Patterson function of its characteristic function, that is $\mathbf{i v}(A)=d^{2}\left(\mathbf{1}_{A}\right)$, all features of interval contents can and will be expressed in terms of Patterson functions. We introduce below the most basic properties of $d^{2}$, which will motivate the ensuing definitions for finitely measured subsets of $G$ that share the same interval contents, and more generally functions in $\Sigma_{\mathrm{C}}(G, K)$ that share the same Patterson function.

Proposition 3.5 (Invariance under transposition and inversion). If $G$ is unimodular, then for every $E \in \Sigma_{\mathrm{C}}(G, K)$, for every $g \in G, d^{2}\left(T_{g}(E)\right)=d^{2}(E)$; furthermore, if $G$ is abelian, then $d^{2}(I(E))=d^{2}(E)$.

Proof. The transposition invariance is implied by the left translation invariance of the Haar measure on $G$ : for every $x \in G, d^{2}\left(T_{g}(E)\right)(x)=\int \overline{E\left(g^{-1} y x^{-1}\right)} E\left(g^{-1} y\right) d \mu(y)=\int \overline{E\left(z x^{-1}\right)} E(z) d \mu(g z)=$ $\int \overline{E\left(z x^{-1}\right)} E(z) d \mu(z)$, where the variable substitution $y=g z$ is made in the second equality.

If $G$ is abelian, the inversion invariance is a consequence of the commutativity of the convolution product and the involutive property of the inversion: $d^{2}(I(E))=I(I(E)) * I(E)=E * I(E)=$ $I(E) * E=d^{2}(E)$.

\footnotetext{
${ }^{7}$ It could be defined for a larger set of functions, e.g. the algebra $L^{1}(\mu)$ of $\mu$-integrable maps from $G$ to $\mathbb{C}$ or the algebra $L^{2}(\mu)$ of maps from $G$ to $\mathbb{C}$ whose square is $\mu$-integrable, but $\Sigma_{\mathbb{C}}(G, k)$ where $k \subset K$ is sufficient for musical applications.
} 


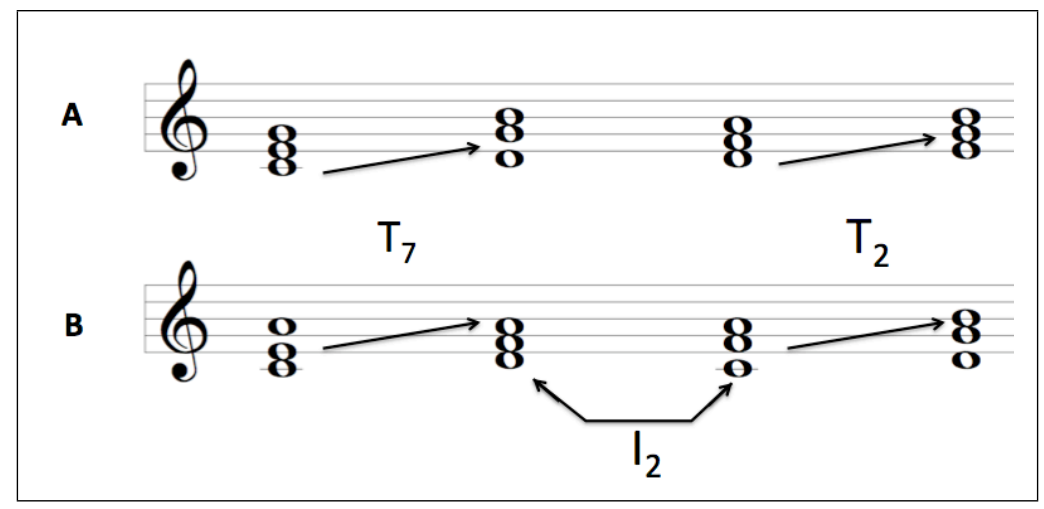

Figure 3: The interval vector changes when $A$ is transformed by $I_{4}$.

The invariance under translation may also hold without the hypothesis that $G$ is unimodular, for instance for $T_{g}$ with $g$ central in $G$, that is for every $h \in G, g h=h g$.

Example 3.6. As a counterexample of the invariance, consider the GIS of major and minor triads with the dihedral group of transpositions and inversions as the interval group with 24 elements, and let for instance $A=\{\{0,4,7\},\{2,7,11\},\{2,5,9\},\{4,7,11\}\}$ and $B=I_{4}(A)$ be its "translate" by the inversion $I_{4}: x \mapsto 4-x$, i.e. $B=\{\{0,4,9\},\{2,5,9\},\{2,7,11\},\{0,5,9\}\}$. We can see in Figure 3 that the inversion $I_{2}: x \mapsto 2-x$ occurs twice in $B$ but never in $A$, i.e. $\mathbf{i v}(B)\left(I_{2}\right)=2$ while $\mathbf{i v}(A)\left(I_{2}\right)=0$. Since every transposition $T_{i}$ is central in $G$, one can check that $\operatorname{iv}\left(T_{i}(A)\right)(g)=\operatorname{iv}(A)(g)$ for all $g \in G$.

\section{Z-relation and homometry}

\subsection{Definitions}

Definition 4.1. The elements of a family $\left(A_{j}\right)_{j \in J}$ valued in $\tilde{\mathcal{A}}$ are said to be Z-related if they have the same interval content almost everywhere. If, in addition, for every distinct $j, k$ in $J$, $\left[A_{j}\right]_{D} \neq\left[A_{k}\right]_{D}$, then the elements of $\left(A_{j}\right)_{j \in J}$ are said to be non-trivially Z-related.

Example 4.2. In $\mathbb{Z}_{8},\{1,2,3,6\}_{8}$ and $\{0,1,3,4\}_{8}$ are non-trivially Z-related. It is the simplest example (with subsets).

Definition 4.3. Let $\left(E_{j}\right)_{j \in J}$ a family of elements of $\Sigma_{\mathcal{C}}(G, K)$. Elements of $\left(E_{j}\right)_{j \in J}$ are said to be homometric if they have the same Patterson function almost everywhere. If, in addition, for every distinct $j, k$ in $J,\left[E_{j}\right]_{\mathcal{D}} \neq\left[E_{k}\right]_{\mathcal{D}}$, the $A_{j}$ are said to be non-trivially homometric.

It should be noted that the Z-relation as defined by Allen Forte in [6, section 1.9] is what we call non-trivial Z-relation, and that our definition of homometry follows Rosenblatt [15]. We choose these definitions so that Z-relation and homometry are equivalence relations on $\tilde{\mathcal{A}}$ and $\Sigma_{\mathrm{C}}(G, K)$, respectively ${ }^{8}$

Obviously, subsets of $\mathcal{A}$ are Z-related if and only if their characteristic functions are homometric.

\footnotetext{
${ }^{8}$ In [1] the equivalence relation is called Lewin's relation, leaving to 'Z-relation' its traditional meaning.
} 


\subsection{Elementary properties}

We will give now properties of the Patterson function related to monotonicity, periodicity and commutation with quotients.

In order to give a monotonicity property of the Patterson function, we introduce a pointwise order on $\Sigma_{\mathrm{C}}(G, \mathbb{R})$ : we note $E \leqslant F$ if, for every $x$ in $G, E(x) \leqslant F(x)$. This order is compatible with the inclusion order on $\tilde{\mathcal{A}}$, i.e. the natural bijection between $\tilde{\mathcal{A}}$ onto $\Sigma_{\mathcal{C}}(G,\{0,1\})$ is an increasing map.

Lemma 4.4. For all distributions $E, F$ in $\Sigma_{\mathcal{C}}\left(G, \mathbb{R}_{+}\right)$, if $E \leqslant F$, then $d^{2}(E) \leqslant d^{2}(F)$, i.e. $d^{2}$ : $\Sigma_{\mathrm{C}}\left(G, \mathbb{R}_{+}\right) \mapsto \Sigma_{\mathrm{C}}\left(G, \mathbb{R}_{+}\right)$is an increasing map. In particular, for every $A, B$ in $\tilde{\mathcal{A}}$, if $A \subset B$ then $\operatorname{iv}(A) \leqslant \operatorname{iv}(B)$.

Proof. For every $x, y$ in $G, 0 \leqslant E(y) \leqslant F(y)$ and $0 \leqslant E\left(y x^{-1}\right) \leqslant F\left(y x^{-1}\right)$, therefore taking the product term by term, $E\left(y x^{-1}\right) E(y) \leqslant F\left(y x^{-1}\right) F(y)$; moreover the Lebesgue integral with measure $\mu$ is positive, so finally $d^{2}(E) \leqslant d^{2}(F)$.

Proposition 4.5. For every distribution $E$ in $\Sigma_{C}(G)$, for $k \in \mathbb{C}, d^{2}(k E)=|k|^{2} d^{2}(E)$. Moreover, if $G$ is commutative, then for all distributions $E, F$ in $\Sigma_{\mathcal{C}}(G) d^{2}(E * F)=d^{2}(E) * d^{2}(F)$.

Proof. The first part of the proposition is obvious. To prove the second part, we assume that $G$ is commutative. Let $E, F \in \Sigma_{\mathrm{C}}(G)$. It is straightforward to see that $I(E * F)=I(E) * I(F)$, so we have $d^{2}(E * F)=I(E * F) * E * F=I(E) * I(F) * E * F$, then the result follows by commutativity of the convolution product.

Proposition 4.6 (Periodicity invariance). Let $E \in \Sigma_{\mathrm{C}}(G)$. If for some $r \in G$, for every $g \in G$, $E\left(g r^{-1}\right)=E(g)$, then for every $g \in G, d^{2}(E)\left(g r^{-1}\right)=d^{2}(E)(g)$.

There is a partial and fuzzy converse result for $\{0,1\}$-valued distributions: if $A \in \tilde{\mathcal{A}}$ has a finite measure and there is $r \in G$ such that $\mathbf{i v}(A)(r)=\mathbf{i v}(A)(e)$, then there are $N, N^{\prime} \mu$-negligible subsets of $G$ such that $A \sqcup N=A r \sqcup N^{\prime}=A \cup A r$, that is, A is "almost periodic".

Proof. $d^{2}(E)\left(g r^{-1}\right)=\int \overline{E\left(h\left(g r^{-1}\right)^{-1}\right)} E(h) d \mu(h)=\int \overline{E\left(h r g^{-1}\right)} E(h) d \mu(h)$, so by right translation invariance of $\mu, d^{2}(E)\left(g r^{-1}\right)=\int \overline{E\left(h^{\prime} g^{-1}\right)} E\left(h^{\prime} r^{-1}\right) d \mu\left(h^{\prime}\right)=\int \overline{E\left(h^{\prime} g^{-1}\right)} E\left(h^{\prime}\right) d \mu\left(h^{\prime}\right)=d^{2}(E)(g)$.

As for the second part of the proposition, we have

$$
\begin{array}{r}
A r=(A \cap A r) \sqcup\left(A^{C} \cap A r\right) \\
A=(A r \cap A) \sqcup\left(A r^{C} \cap A\right) \\
A \sqcup\left(A^{c} \cap A r\right)=A \cup A r=A r \sqcup\left(A r^{C} \cap A\right)
\end{array}
$$

By right translation invariance of $\mu, \mu(A r)=\mu(A)$, so by (1), $\mu(A)=\mu(A \cap A r)+\mu\left(A^{C} \cap A r\right)$; moreover, $\mu(A)=\operatorname{iv}(A)(e)=\operatorname{iv}(A)\left(r^{-1}\right)=\operatorname{iv}(A)(r)=\mu(A \cap A r)$ is finite, so $\mu\left(A^{C} \cap A r\right)=0$, so $N:=A^{c} \cap A r$ is negligible. In a similar way, we get from (2) that $N^{\prime}:=A r^{C} \cap A$ is negligible. We finally get the result by (3).

Example 4.7. The Proposition 4.6 tells us that any periodic distribution has a periodic interval content. Hence the interval content of any of Messiaen's modes of limited transposition will be periodic. For example - see Figure 4 - the interval vector of $A=\{0,1,3,6,7,9\}_{12}$ is $\operatorname{iv}(A)=$ $[6,2,2,4,2,2,6,2,2,4,2,2]$. Since $T_{6}(A)=A$, we have $T_{6}(\mathbf{i v}(A))=\mathbf{i v}(A)$.

We will make use of the following simple necessary condition on measure equality for Z-relation. 


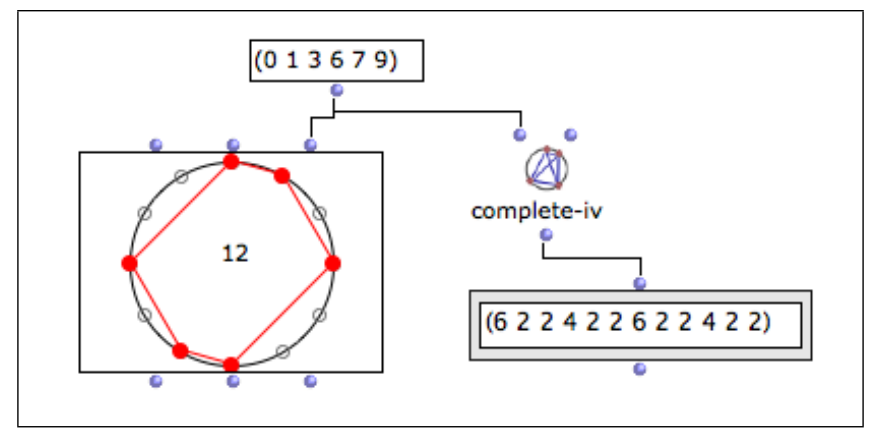

Figure 4: An OpenMusic patch showing that the interval vector of a periodic set is periodic.

Lemma 4.8. If $\left(A_{j}\right)_{j \in J}$ is a family of Z-related subsets of $G$, then all the $A_{j}$ have the same measure. Proof. For every $j \in J, \mu\left(A_{j}\right)=\mathbf{i v}\left(A_{j}\right)(e)$, where $e$ is the neutral element of $G$.

In particular, if the topology on $G$ is discrete, then any two Z-related subsets of $G$ have the same cardinality.

\section{$5 \quad$ Interval structure and interval content}

We will now build a link between interval content and interval structure, expressing the former using the latter. We will focus our attention to a restricted class of discrete groups, namely discrete groups with a total order compatible with left translation.

Definition 5.1. An left-(totally-)ordered group is a couple $(G, \leqslant)$ where $G$ is a discrete group and $\leqslant$ is a total order on $G$ which is compatible with left translation, that is for every $f, g, h$ in $G$, if $f \leqslant g$ then $h f \leqslant h g$.

Examples of left-ordered groups are all abelian ordered groups, e.g. $\mathbb{Z}, \mathbb{R}$, and the time spans group $\mathbb{R} \rtimes_{m} \mathbb{R}_{+}^{*}$ fitted with Lewin's attack order, which is simply the lexicographic order associated with the usual order on $\mathbb{R}$ and $\mathbb{R}_{+}^{*}$. Every direct product of left-ordered groups fitted with the lexicographic order associated to the orders of these groups is a left-ordered group too.

Definition 5.2. Let $G$ be a left-ordered group. For every finite subset $A$ of $G$, there is a unique strictly increasing family $\left(a_{i}\right)_{i \in \llbracket 1, n \rrbracket}$ where $n=|A|$, such that $A=\left\{a_{i}\right\}_{i \in \llbracket 1, n \rrbracket}$. The interval structure of $A$ is the family is $(A)=\left(\operatorname{int}\left(a_{i}, a_{i+1}\right)\right)_{i \in \llbracket 1, n-1 \rrbracket}$.

Example 5.3. Let $A=\{-3,-1,1,5,6\}$ in $\mathbb{Z} ;$ is $(A)=(2,2,4,1)$. Let $B=\left\{(2,1),(3,1),(5,2),\left(7, \frac{1}{2}\right),(7+\right.$ $\left.\left.\frac{1}{2}, \frac{1}{2}\right),(9,3)\right\}$ in the time spans group $\mathbb{R} \rtimes_{m} \mathbb{R}_{+}^{*} ;$ is $(B)=\left((1,1),(2,2),\left(1, \frac{1}{4}\right),(1,1),(3,6)\right)$.

Proposition 5.4. Let $G$ be a left-ordered group. The interval structure of every finite subset of $G$ is invariant by left translation, that is for every finite subset $A$ of $G$, for every $g$ in $G$, is $(g A)=\operatorname{is}(A)$. Conversely, if $A, B$ are finite subsets of $G$ such that is $(A)=\operatorname{is}(B)$, then there is $g \in G$ such that $B=g A$. 
Proof. The invariance of interval structure by left translation directly follows from the preservation of intervals by left translation. As for the second part of the proposition, it is obvious that by defining $g=\min (B) \min (A)^{-1}$ we get by finite induction on the lists defined by ordering $A$ and $B$ that $B=g A$.

We shall now define a partition of a non-negative element of a left-ordered group, which naturally generalizes the notion of partition of a positive integer, and a consecutive subfamily of a sequence valued in a left-ordered group.

Definition 5.5. Let $G$ be a left-ordered group, let $e$ be the neutral element of $G$, let $p \in G$ such that $p \geqslant e$. An ordered partition of $p$ is a family of elements of $G\left(d_{j}\right)_{j \in \llbracket 1, k \rrbracket}$ such that $k \in \mathbb{N}$, for all $j$ in $\llbracket 1, k \rrbracket d_{j}>e$ and $\prod_{j=1}^{k} d_{j}=p$.

Definition 5.6. Let $G$ be a left-ordered group, let $A=\left(a_{j}\right)_{j \in \llbracket 1, k \rrbracket}$ be a family of elements of $G$. A consecutively-indexed subfamily of $A$ is any subfamily $\left(a_{j}\right)_{j \in J}$ of $A$ such that $J=\llbracket l, m \rrbracket$ with $1 \leqslant l \leqslant m \leqslant k$.

Theorem 5.7. Let $A=\left\{a_{i}\right\}_{i \in \llbracket 1, k \rrbracket}$ be a finite subset of a left-ordered group $G$, such that $\left(a_{i}\right)_{i}$ is strictly increasing. We denote by $\left(d_{i}\right)_{i \in \llbracket 1, k-1 \rrbracket}$ the interval structure of $A$. For every $p \in G$, let $\mathcal{I}_{p}(A)=\left\{\left(j, j^{\prime}\right) \in \llbracket 1, k-1 \rrbracket^{2}, j+1 \leqslant j^{\prime}\right.$ and $\left.\prod_{i=j}^{j^{\prime}} d_{i}=|p|\right\}$, where $|p|=\max \left(p, p^{-1}\right)$; then $\operatorname{iv}(A)(p)=\#\left(\mathcal{I}_{p}(A)\right)$, that is, $\mathbf{i v}(A)(p)$ is equal to the number of consecutively-indexed subfamilies of is $(A)$ which are partitions of $|p|$.

Proof. For every $p \in G \backslash\{e\}, \operatorname{iv}(A)(p)=\mathbf{i v}(A)(|p|)$, so we can suppose that $p \geqslant e$. The map

$$
\begin{aligned}
\mathcal{I}_{p}(A) & \rightarrow A \cap A p \\
\left(j, j^{\prime}\right) & \mapsto a_{j^{\prime}}=a_{j} p
\end{aligned}
$$

is well-defined and bijective, and $\#(A \cap A p)=\mathbf{i v}(A)(p)$.

This theorem may be used to compute the interval content from an interval structure. For instance, the time spans group $G$ is non-commutative and has no central element besides the neutral $(0,1)$, so interval structure and the interval content have exactly the same invariance properties on this group, including invariance by left translation. Thus, an approach for finding Z-related subsets of the time spans is by generating interval structures and sorting them by their interval content. For example, by taking $\mathcal{E}=\prod_{j=1}^{4}\left\{\left(1+\frac{k}{2}, 2^{l}\right)\right\}_{k=0, \ldots, 6, l=-1,0,1}$, we find with computer search two and only two interval structures in $\mathcal{E}$ that have the same interval content, and by "integrating them", we obtain that the time spans sets $\left\{(0,1),(1,1),\left(2, \frac{1}{2}\right),\left(\frac{5}{2}, \frac{1}{2}\right),\left(\frac{7}{2}, \frac{1}{4}\right)\right\},\left\{(0,1),(1,1),\left(\frac{5}{2}, \frac{1}{2}\right),\left(3, \frac{1}{2}\right),\left(\frac{7}{2}, \frac{1}{4}\right)\right\}$ are Z-related, as shown in Figure 5.

\section{Patterson function transfer through quotients}

We keep the same notations as in the previous section. Let $H$ be a closed and normal subgroup of $G$; then $G / H$ is a locally compact group. Details and proofs for the measure theory results below can be found in [14, Chap. 3, 3.3(i) and 4.5].

Let $\mu$ be a right Haar measure on $G, \nu$ a right Haar measure on $H$ with the topology induced by $G$, and $\lambda$ the unique right Haar measure on $G / H$ such that for every $E$ in $\Sigma_{\mathrm{C}}(G)$

$$
\int_{G / H} \int_{H} E(h x) d \nu(h) d \lambda\left([x]_{H}\right)=\int_{G} E d \mu
$$




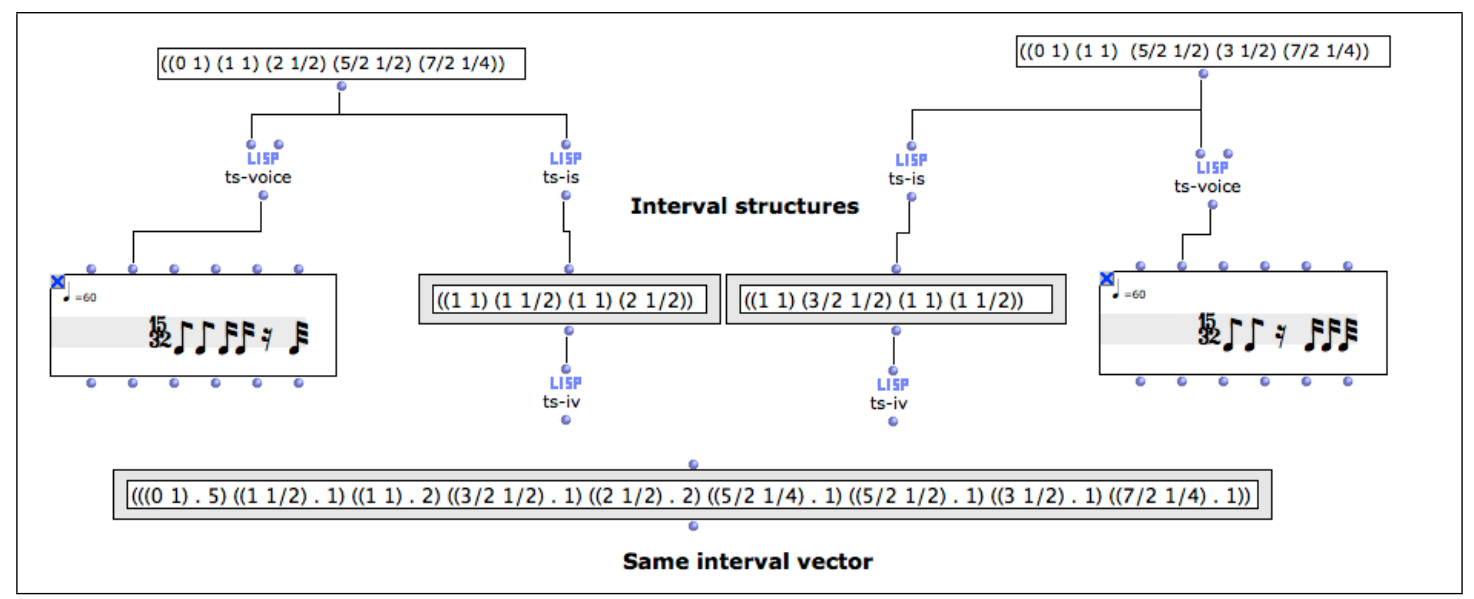

Figure 5: Example of Z-relation between two time spans (non-commutative case).

$$
\text { By defining } \quad \begin{aligned}
\sim & : \Sigma_{\mathrm{C}}(G) \\
E & \rightarrow \Sigma_{\mathrm{C}}(G / H) \\
E & \mapsto \widetilde{E}:[x]_{H} \mapsto \int_{H} E(h x) d \nu(h)
\end{aligned}
$$

the equality above is rewritten $\int \widetilde{E} d \lambda=\int E d \mu$.

In the particular case of $G=\mathbb{Z}$ with the discrete topology, let $H$ be a non-trivial subgroup of $\mathbb{Z}$ : $H=n \mathbb{Z}$ for some integer $n>1$; for all $E \in \Sigma_{\mathcal{C}}(\mathbb{Z}), k \in \mathbb{Z}, E([k])=\sum_{j \in \mathbb{Z}} E(j n+k)$.

Theorem 6.1. With the previous hypotheses and notations, the $\sim$ operator defined above and the Patterson function operator "commute", that is, for every $E \in \Sigma_{\mathcal{C}}(G), d^{2}(\widetilde{E})=\widetilde{d^{2}(E)}$ :

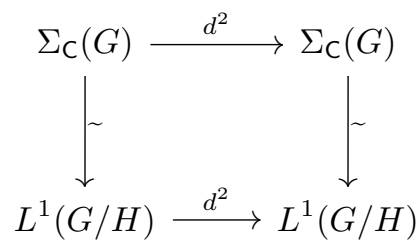

Proof. We reuse two results of [14, Chap. 3, 5.3], namely that ${ }^{\sim}: \Sigma_{\mathcal{C}}(G) \rightarrow \Sigma_{\mathcal{C}}(G / H)$ is a morphism of algebras with the convolution product, and that $I$ and $\sim$ commute. Thus, for every $E \in \Sigma_{\mathrm{C}}(G)$, $d^{2}(\widetilde{E})=I(\widetilde{E}) * \widetilde{E}=\widetilde{I(E)} * \widetilde{E}=I \widetilde{(E) * E}=\widetilde{d^{2}(E)}$.

Corollary 6.2. Under the same notations and hypotheses as the previous theorem, if $E_{1}, \ldots, E_{s}$ in $\Sigma_{\mathrm{C}}(G)$ are homometric, then $\widetilde{E_{1}}, \ldots, \widetilde{E_{s}}$ are homometric in $\Sigma_{\mathrm{C}}(G / H)$.

Example 6.3. $A=\{0,1,2,6,8,11\}$ and $B=\{0,1,6,7,9,11\}$ are Z-related in $\mathbb{Z}$, so their projections $\pi(A)=\{0,1,2,6,8,11\}_{12}$ and $\pi(B)=\{0,1,6,7,9,11\}_{12}$ are Z-related in $\mathbb{Z}_{12}$. Actually, the projections $\{0,1,2,6,8,11\}_{n}$ and $\{0,1,6,7,9,11\}_{n}$ are homometric for every $n \in \mathbb{N}, n \geqslant 2$; and they collapse into multisets for $n \leqslant 11$.

Example 6.4. In general, non-triviality is not preserved through quotients. The sets $A=\{0,1,2,3,4,6,7,8,11\}$ and $B=\{0,1,4,5,6,7,8,9,11\}$ are Z-related in $\mathbb{Z}$, and so are their projections on $\mathbb{Z}_{12}$; however, 
these projections are related by transposition, namely $\pi(B)=T_{5}(\pi(A))$. It is easy to see that for any Z-relation of subsets of $\mathbb{Z}$ one can always find a $n^{\prime}$ such that for every $n \geqslant n^{\prime}$ the non-triviality of a Z-relation is preserved $\bmod n$. In this case, $n^{\prime}=13$ is enough: this follows from the fact that for $n \geqslant n^{\prime}$, in $B \bmod n$ there are six consecutive integers, a feature invariant under transposition and inversion, while there is no such configuration in $A \bmod n$.

A loose but always valid choice for $n^{\prime}$ is $n^{\prime}=2(\max (A)-\min (A))=2(\max (B)-\min (B))$.

Note that the converse of Corollary 6.2 is not true: $A=\{0,1,2,5\}_{8}$ and $B=\{3,4,6,7\}_{8}$ are Z-related in $\mathbb{Z}_{8}$, but for every $A^{\prime}, B^{\prime}$ subsets of $\mathbb{Z}$ such that $\pi\left(A^{\prime}\right)=A$ and $\pi\left(B^{\prime}\right)=B$, it is easy to see that $\operatorname{diam}\left(A^{\prime}\right) \neq \operatorname{diam}\left(B^{\prime}\right)$, where diam denotes the diameter, hence $A^{\prime}$ and $B^{\prime}$ are not Z-related.

\section{The hexachord theorem}

\subsection{Patterson functions of generalized hexachords}

The hexachord theorem has been significantly popular in the literature - see [13, Chap. V, 5.16], [11, Sec. 6.6] and [3. Since it is actually a feature of Patterson functions, we propose here a restatement in the framework of locally compact (not necessarily commutative) GIS, and add a few geometric remarks.

$G, \mathcal{A}, \mu$ are defined as above. We will additionally assume in this subsection that $\mu(G)$ is finite, which is equivalent to the compactness of $G$.

The initial form of the hexachord theorem by Milton Babbitt is an invariance property of the interval vector by complementation. Wherever there is no ambiguity, $\mathbf{1}_{G}$ will be writter 9 , and for every $a \in \mathbb{C}, a \mathbf{1}_{G}$ will be written $a$. For every measurable subset $A \subset G, \mathbf{1}_{A^{C}}=1-\mathbf{1}_{A}$, where $A^{C}=G \backslash A$, hence we can naturally extend the complement function to $\Sigma_{\mathrm{C}}(G)$, which we define as $C: E \mapsto 1-E$. This extension allows us to express a generalization of the hexachord theorem, which results immediately from the following lemma.

Lemma 7.1. For every $E$ in $\Sigma_{\mathcal{C}}(G)$, for every $a \in \mathbb{R}, d^{2}(a-E)=a^{2} \mu(G)-2 a \operatorname{Re}\left(\int E d \mu\right)+d^{2}(E)$. In particular, for $a=1, d^{2}(C(E))=\mu(G)-2 \operatorname{Re}\left(\int E d \mu\right)+d^{2}(E)$.

Proof. The inversion $I$ is linear and $I(a)=\bar{a}=a$, so $d^{2}(a-E)=I(a-E) *(a-E)=a * a-a * E-$ $I(E) * a+I(E) * E=a^{2} \mu(G)-a \int E d \mu-a \int \bar{E} d \mu+d^{2}(E)=a^{2} \mu(G)-2 a \operatorname{Re}\left(\int E d \mu\right)+d^{2}(E)$.

Theorem 7.2 (Generalized hexachord theorem). For every $E$ in $\Sigma_{\mathrm{C}}(G), d^{2}(C(E))=d^{2}(E)$ if and only if $\operatorname{Re}\left(\int E d \mu\right)=\mu(G) / 2$.

In the non-commutative case, this theorem admits two versions, i.e. it holds with either left or right interval content.

From a geometric point of view, $C$ is the central symmetry relative to constant map $1 / 2$; this means that the hexachord theorem is a condition of invariance of the Patterson function under this kind of symmetry - see Figure 6 - just like its invariance under $I$, but that is valid only under some normalization condition. If $E$ is a $\{0,1\}$-valued map, i.e. $E$ is the characteristic map of a measurable set $A \subset G$, this normalization condition requires that $\mu(A)=\mu(G) / 2$, which in the case where $G$ is discrete means that the cardinality of $A$ is half the cardinality of $G$, which is already the original result.

\footnotetext{
${ }^{9}$ All the more so since without loss of generality, one can assume $\mu(G)=1$.
} 


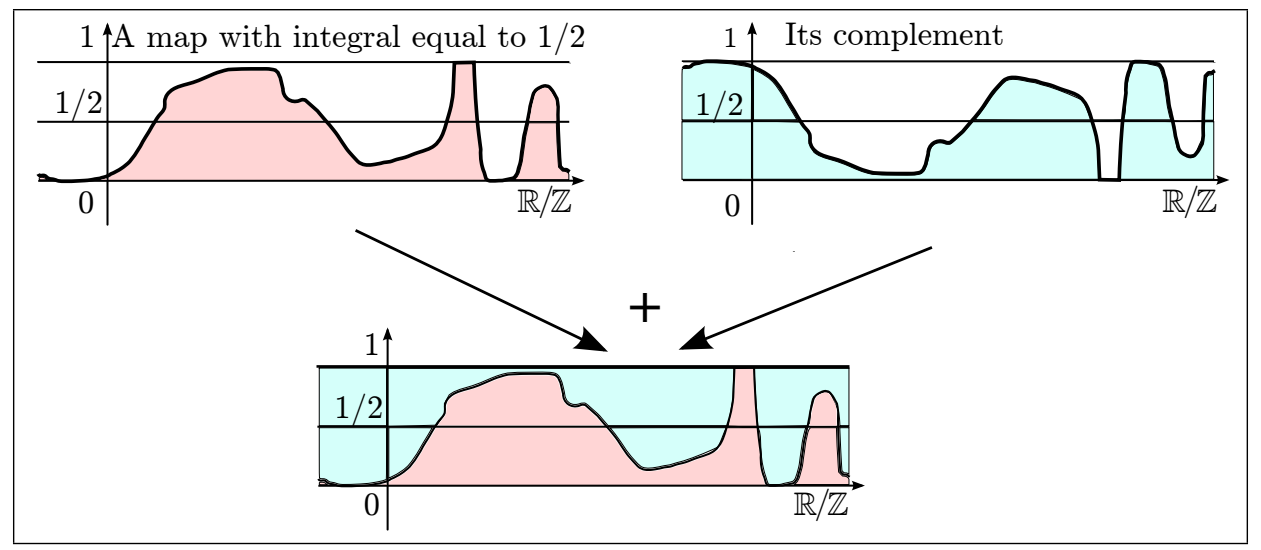

Figure 6: An illustration of the generalized hexachord theorem in the case of $G=\mathbb{R} / \mathbb{Z}$.

A more general formulation of the hexachord theorem - see [13], is by computing the difference between the interval contents of a function and of its complement. It entails immediately that homometry is preserved by the complement operator $C$.

Corollary 7.3. For every $E$ in $\Sigma_{\mathrm{C}}(G), d^{2}(E)-d^{2}(C(E))$ is a constant map.

Proof. This results immediately from Lemma 7.1

Corollary 7.4. For every $E_{1}, \ldots, E_{s}$ in $\Sigma_{\mathcal{C}}(G), E_{1}, \ldots, E_{s}$ are homometric if and only if $C\left(E_{1}\right), \ldots, C\left(E_{s}\right)$ are homometric.

A previous generalization of Babbitt's hexachord theorem to the unit circle is the subject of [3], but it cannot be further generalized for lack of reference to an integration theory and generalized notion of interval. Nevertheless, the paper mentions the problem of an hexachord theorem on the sphere $S^{2}$; unfortunately, since there is no topological group structure on the sphere $S^{2}$ (with its usual topology), the notions of interval and interval content in a Generalized Interval System are meaningless 10

\subsection{Some examples of the generalized hexachord theorem}

- Musical scales can be modelized as elements of a torus, which is the space of a GIS under transposition. Say we define the set of 'in tune' scales as major scales whose maximal deviation from a well-tempered major scale does not exceed 10 cents, e.g. the 'in tune' D major scales would be in $[190,210] \times[390,410] \times[590,610] \times[690,710] \times[890,910] \times[1090,1110] \times[90,110]$ where each pc is given in cents. So the reunion ITS of all 12 'in tune' major scales is a subset of the torus $\mathbb{T}^{7}=(\mathbb{R} / 1200 \mathbb{Z})^{7}$, with measure $1 / 60^{7}$ of the whole torus. Now the complement OTS (Out of Tune Scales) has the same interval content, up to a constant.

\footnotetext{
${ }^{10}$ Only the spheres $S^{1}$ (the circle), $S^{3}$ (in dimension 4), and in some measure $S^{7}$ may be provided with a group structure and a Haar measure compatible with their natural topology. It is conceivable that a more general notion of interval could be defined as geodesics on manifolds.
} 
- We have explained why, for lack of a group structure, we cannot hope to give a hexachord theorem in the sphere $S^{2}$. But in 4 dimensions, the sphere $S^{3}$ is a compact Lie group, for instance one can set $G=S U(2)=S^{3}$ :

Definition 7.5. The group $S U(2)$ is the set of complex matrices $\left(\begin{array}{cc}z_{1} & -z_{2} \\ z_{2} & \bar{z}_{1}\end{array}\right)$ with determinant 1. As a set it coincides with the sphere in $\mathbb{C}^{2}:\left\{\left|z_{1}\right|^{2}+\left|z_{2}\right|^{2}=1\right\}$, e.g. the sphere $S^{3}$ in $\mathbb{R}^{4}$.

The group operation is then simply matrix multiplication. It can be shown that, parametrizing $S^{3}$ with $z_{1}=\cos \theta e^{i \phi}, z_{2}=\sin \theta e^{i \psi}$ with $\theta \in[0, \pi / 2], 0 \leq \phi, \psi \leq 2 \pi$, the Haar measure is (up to a constant) $\mu_{1}=\sin 2 \theta d \theta d \phi d \psi$.

With this measure, the hexachord theorem with either right or left interval content hold on $S^{3}$. This may have interesting applications in visualization of musical structures on this hypersphere, see for instance [4].

- We can now turn back to discrete, but non abelian, groups. The Haar measure is the counting measure. For instance, let $G$ be the dihedral group over $\mathbb{Z}_{12}$, which makes a GIS for instance on the space of major and minor triads. A very simple 'hexachord' is the set $M$ of major triads. It is a copy of the normal subgroup $T$ of transpositions. The interval vector on $M$ (or $T$, if $G$ acts on itself) is computed immediately with the following general proposition:

Proposition 7.6. Let $H$ be a subgroup of $G$. Then

$$
{ }^{l} \mathbf{i v}(H)(g)={ }^{r} \mathbf{i v}(H)(g)=\left\{\begin{array}{ll}
\mu(H) & \text { when } g \in H \\
0 & \text { else }
\end{array} .\right.
$$

Our generalized hexachord theorem now states that the complement of $M$ (i.e. the minor triads) share the same interval vector. More generally, there are as many transformations (intervals) between a given triad and the major triads, as there are between this triad and the minor triads.

For a less trivial case, consider for instance the $\langle L P R>$ group of the (dual) neo-Riemannian Tonnetz, acting as interval group on the same set of major and minor triads. If $A=$ white triads (the 6 triads without black keys, $C E G, D F A \ldots A C E$ ) and $B$ is its complement (triads with at least one black key), then

1. In $A$ there is 6 times the 'interval' $R$, meaning 3 pairs of relative major-minor triads. The theorem yields that there are $6+12$ cases of $R$ in $B$, i.e. the 9 remaining pairs of relative triads.

2. Less obviously, there are no cases of the transformation $R P$ (which moves any major triad to its translate by a major third) in $A$, hence, without further ado, there are 12 occurences of $R P$ in $B$ (e.g. E major to $\mathrm{G} \#$ major).

For more examples, see [2]. 


\section{Conclusion}

We have extended and unified the definition of interval content and Patterson function to a larger framework using common mathematical tools, namely Haar measures and Lebesgue integration theory. This approach has allowed us to obtain the following results on Patterson functions, also valid in the non-commutative case:

- translation and periodicity invariance;

- transfer through quotients;

- a generalization of the hexachord theorem to a large class of GIS;

- first musical examples of Z-relation in a non-commutative GIS.

In our next paper, Discrete Phase Retrieval in Musical Distributions [12, we tackle the more general question of searching for all possible distributions yielding a given Patterson function, a general formulation of the search for sets of a musical parameter with a given interval vector, i.e. of all Z-related sets.

\section{Acknowledgments}

The revision of the manuscript benefited crucially from the details comments and critiques provided by the two anonymous reviewers to which we are immensely grateful. Special thanks go to our colleague Pierre Beauguitte for having carefully proofread old versions of the two articles and suggested many pertinent mathematical corrections.

\section{References}

[1] Emmanuel Amiot. Eine Kleine Fourier Musik. In Mathematics and Computation in Music, number 37-3 in Communications in Computer and Information Science, pages 469-476, Berlin, 2007. SMCM, Springer. Available from: http://www.springerlink.com/content/ rv46154m2n668630/.

[2] Emmanuel Amiot. Two hexachordal theorems in general compact groups. preprint, 2009. Available from: http://canonsrythmiques.free.fr/pdf/twoHexachordThms.pdf.

[3] Brad Ballinger, Nadia Benbernou, Francisco Gomez, Joseph O'Rourke, and Godfried Toussaint. The Continuous Hexachoral Theorem. In E. Chew, A. Childs, and C.-H. Chuan, editors, Matematics and Computation in Music, volume 38 of Communications in Computer and Information Science, pages 11-21, New Haven, CT, USA, 22 June 2009. SMCM, Springer.

[4] Gilles Baroin. The Planet-4D Model: an Original Hypersymmetric Music Space Based on Graph Theory. In Mathematics and Computation in Music, Third International Conference, number 6276 in LNAI, pages 326-329, Paris, 2011. SMCM, Springer. Available from: http: //www.springerlink. com/content/qq67v01w208k1453/

[5] Michael Buchler. Relative Saturation of Subsets and Interval Cycles as a Means for Determining Set-Class Similarity. PhD thesis, University of Rochester, 1997. Available from: http://myweb. fsu.edu/mbuchler/dissertation/dissertation.html. 
[6] Allen Forte. The Structure of Atonal Music. Yale University Press, second edition, 1977.

[7] Daniele Ghisi. From z-relation to homometry: an introduction and some musical examples. Paper delivered at the MaMuX Seminar December, 12, IRCAM, 2010. Available from: http:// repmus.ircam.fr/_media/mamux/saisons/saison10-2010-2011/ghisi-2010-12-12.pdf

[8] Howard Hanson. Harmonic Materials of Modern Music. Appleton-Century-Crofts, 1960.

[9] Oren Kolman. Transfer Principles for Generalized Interval Systems. Perspectives of New Music, 42(1):150-190, 2004.

[10] David Lewin. Intervallic Relations Between Two Collections of Notes. J. Music Theory, 3(2), 1959.

[11] David Lewin. Generalized Musical Intervals and Transformations. Yale University Press, second edition by Oxford University Press, 2007 edition, 1987.

[12] John Mandereau, Daniele Ghisi, Emmanuel Amiot, Moreno Andreatta, and Carlos Agon. Discrete Phase Retrieval in Musical Distributions. J. Math. ES Music, 5, 2011.

[13] John Rahn. Basic Atonal Theory. Schirmer Books, 1980.

[14] Hans Reiter. Classical Harmonic Analysis and Locally Compact Groups. Oxford University Press, 1968.

[15] Joseph Rosenblatt. Phase Retrieval. Comm. Math. Phys., 95:317-343, 1984.

[16] Walter Rudin. Real and Complex Analysis. McGraw-Hill, third edition, 1987.

[17] Dan Tudor Vuza. Some Mathematical Aspects of David Lewin's Book: Generalized Musical Intervals and Transformations. Perspectives of New Music, 26(1), 1988. 